\title{
Conocimiento como medio para la interacción de alumnos de primer grado de educación primaria con las fracciones
}

\section{Knowledge as a means of interacting primary school freshmen with fractions}

\author{
HERNÁNDEZ-GUTIÉRREZ, Francisco Javier*† \& ROSALES-POSADA, Norma Susana \\ Escuela Normal Rural “Gral. Matías Ramos Santos” Secretaría de Educación del Estado de Zacatecas. Av José Santos \\ Valdéz 6, 98820 San Marcos, Zac.
}

ID $1^{\text {er }}$ Autor: Francisco Javier, Hernández-Gutiérrez / ORC ID: 0000-0002-4134-134, Researcher ID: L-2003-2017, CVU CONACYT ID: 428448

ID $1^{\text {er }}$ Coautor: Norma Susana, Rosales-Posada / ORC ID: 0000-0003-4028-2084, CVU CONACYT ID: 1011449

DOI: $10.35429 / J B E .2019 .8 .3 .1 .8$

Recibido 10 de Abril, 2019; Aceptado 27 de Junio, 2019

\begin{abstract}
Resumen
El objetivo del presente trabajo es identificar los subdominios del conocimiento especializado del profesor de matemáticas (MTSK) en la práctica docente en una sesión-clase de matemáticas, utilizando una perspectiva cualitativa con observación, transcripción y su respectivo análisis, para contribuir a la mejora profesional de los docentes de educación primaria. Al mismo tiempo recabar evidencia desde una Situación Didáctica sobre el impacto que genera un acercamiento de alumnos de primer grado de primaria con el campo de estudio de fracciones, ya que es un contenido no incorporado en el currículo oficial para primer ciclo de primaria. Los resultados del estudio presentan la posibilidad concreta de potencializar contenidos específicos de fracciones en los primeros grados de la educación primaria, un aspecto trascendente en la visualización de este tipo de contenidos en estos primeros grados de educación.
\end{abstract}

Conocimiento especializado del profesor de matemáticas, Teoría de las Situaciones Didácticas, Fracciones

\begin{abstract}
The objective of this paper is to identify the subdomains of Mathematic Teacher Specialized Knowledge (MTSK) in the teaching practice in a mathematics class-session, using a qualitative perspective with observation, transcription and their respective analysis, to contribute to professional improvement of elementary school teachers. At the same time, gathering evidence from a Didactic Situation on the impact generated by an approach of first grade students with the field of fractions study, since it is a content not incorporated in the official curriculum for the first cycle of elementary school. The results of the study present the concrete possibility of potentializing specific contents of fractions in the first grades of elementary education, a transcendent aspect in the visualization of this type of content in these first grades of education.
\end{abstract}

Mathematic Teacher Specialized Knowledge, Theory of Teaching Situations, Fractions

Citación: HERNÁNDEZ-GUTIÉRREZ, Francisco Javier \& ROSALES-POSADA, Norma Susana. Conocimiento como medio para la interacción de alumnos de primer grado de educación primaria con las fracciones. Revista de Educación Básica. 2019. 3-8: 1-8.

*Correspondencia al Autor (Correo Electrónico: frajaher_79@ @otmail.com)

$\dagger$ Investigador contribuyendo como primer autor. 


\section{Introducción}

Ser agente directo en la educación, conduce a mantener una constante reflexión acerca de las acciones que se realizan en contraste con los resultados que se obtienen, es por ello que el análisis de la práctica es un recurso indispensable para hacer una valoración objetiva de las acciones, así mismo reconocer debilidades y fortalezas en el conocimiento que da pauta a las relaciones del proceso enseñanzaaprendizaje. El presente análisis se centra en identificar relaciones del conocimiento del profesor que enseña matemáticas a partir del modelo MTSK (Carrillo, Montes, Contreras, \& Climent, 2017) en una sesión de clases referente al tema de fracciones con alumnos de primer grado de primaria, son importantes los resultados que se evidencian, ya que el contenido abordado no se encuentra actualmente en el currículo oficial. El seguimiento de análisis se da a partir de la estructura de la Teoría de las Situaciones Didácticas TSD (Brousseau, 2007) con una valoración de lo sucedido en cada una de las fases: relaciones Maestra (Ma), Alumnos (Aos), presentes en los subdominios del modelo MTSK y el impacto en los alumnos.

\section{Marco teórico}

El conocimiento especializado que posee un profesor es proporcional al conocimiento que puede construir en sus estudiantes. En este sentido, es preciso hablar del modelo del MTSK (Matematics Teacher's Specialized Knowledge) el conocimiento especializado del profesor de matemáticas presentado en la Figura 1 (Carrillo, Montes, Contreras \& Climent, 2017).

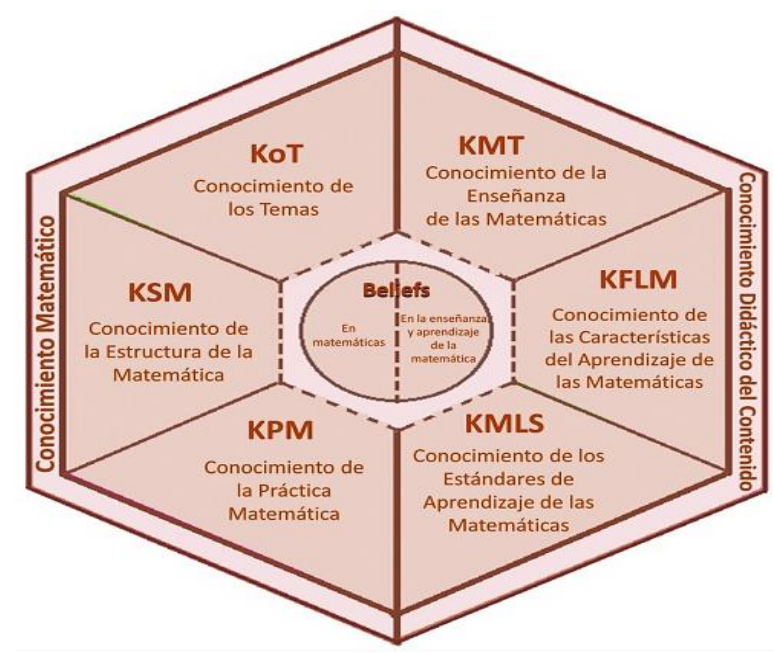

Figura 1 Modelo MTSK

Fuente Carrillo, Montes, Contreras \& Climent 2017

ISSN-2523-2452

ECORFAN $^{\circledR}$ Todos los derechos reservados
El modelo se enfoca al conocimiento que posee el maestro en el campo de las matemáticas; se divide en dos dominios: Conocimiento Matemático (MK) y Conocimiento Didáctico del Contenido (PCK) y tres subdominios en cada uno de los dominios, integra creencias y concepciones sobre la enseñanza y aprendizaje de la disciplina. Analizar la intervención con base al presente modelo, hace necesario analizar cada uno de los subdominios.

\section{Conocimiento Matemático (MK):}

- Conocimiento de lo Temas (KoT): aspectos de los conceptos, contextualización, significados, propiedades.

- Conocimiento de la Estructura de la Matemática (KSM): conexiones transversales, complejización.

- Conocimiento de Práctica Matemática (KPM): Diferentes formas de proceder en la clase, formas de demostrar, procesos de resolución. (PCK):

Conocimiento Didáctico del Contenido

- Conocimiento de las Características del Aprendizaje de las Matemáticas (KFLM): teorías de aprendizaje, cómo aprenden, dificultades, aptitudes.

- Conocimiento de los Estándares de Aprendizaje de las Matemáticas (KMLS): currículo oficial.

- Conocimiento de la Enseñanza de las Matemáticas (KMT): teorías de enseñanza, estrategias, recursos.

Dentro del subdominio KMT, podemos encontrar posturas teóricas complementarias de apoyo para su mejor análisis, como la Teoría de las Situaciones Didácticas (TSD) que caracteriza (Brousseau, 2007) con las situaciones:

- Acción donde los alumnos actúan sobre un medio, se da lugar a momentos a-didácticos que se caracterizan porque el docente no interviene directamente ayudando a encontrar soluciones. 
- Formulación: entre alumnos se emiten mensajes que generan acción a partir de la comprensión de éste.

- Validación: entre alumnos se ponen de acuerdo sobre la verdad o falsedad aseveraciones, son sometidas a la consideración del otro grupo, que debe tener la capacidad de aceptarlas, rechazarlas, pedir pruebas o poner otras aserciones.

- Institucionalización: Propia del docente, conclusiones, recapitular, sistematizar, ordenar, vincular lo que se produjo en diferentes momentos del desarrollo de la secuencia didáctica. Establecer relaciones entre las producciones de los alumnos y el saber cultural.

Desde la Teoría de las Situaciones Didácticas se trabajan contenidos matemáticos, entre ellos fracciones que es un área con deficiencias académicas; (Linares \& Sánchez, 1997) quizá el vago uso social de las fracciones, es consecuencia del poco dominio teórico y didáctico que se tiene de dicho contenido al momento de su enseñanza.

Una de las representaciones con que inicia el currículo formal en la enseñanza de las fracciones es en el marco del subconstructo "parte-todo" que se refiere a situaciones donde un todo o unidad de divide en partes iguales.

El currículo oficial incorpora aprendizajes esperados del campo de fracciones a partir del segundo ciclo de primaria, específicamente en el tema número: "Usa fracciones con denominador hasta 12 para expresar relaciones parte-todo, medidas y resultados de reparto", en la misma línea dentro del tema adición y sustracción: "Resuelve problemas de suma y resta de fracciones con el mismo denominador" (SEP, 2017, pág. 236). Posteriormente en el tercer ciclo, se trabaja la lectura, escritura y orden de números fraccionarios, resolución de problemas de adición y sustracción con denominador múltiplo, así mismo se adentra en la multiplicación y división de fracciones.
El conocimiento necesario que expone el modelo MTSK: estrategias de enseñanza, características de aprendizaje de los alumnos, estándares curriculares, entre otros, empleados y puestos en práctica de forma adecuada, puede llevar al logro de resultados gratificantes en el campo de las fracciones, sobre todo puede determinar marcos pertinentes de análisis de la realidad en la enseñanza de las fracciones para posibilitar las transformaciones en el conocimiento disciplinar y didáctico de los profesores de educación primaria.

\section{Metodología}

El análisis de la práctica también es una herramienta de gran importancia para el trabajo de los docentes, ya que reflexionar sobre lo que sucede en el aula, ya sean aspectos positivos o negativos, brinda la oportunidad de realizar ajustes en las estrategias que se implementan, para mejorar las acciones que se llevan a cabo y por consiguiente los resultados que arrojan los estudiantes. En este sentido (Perales, 2006, pág. 14) argumenta que "el proceso de formación de maestros busca ampliar y hacer más complejos los significados que cada sujeto tiene sobre su práctica", los maestros están en formación continua, pues siempre habrá cosas por mejorar. La experiencia, pero sobre todo la reflexión sobre ella conforma el avance y la mejora no sólo del profesor en lo individual, sino de los alumnos a quienes atiende, así como de la educación y la sociedad.

Este análisis tiene por objetivo identificar la presencia de conocimiento especializado desde el modelo MTSK en la profesora durante el desarrollo de una sesiónclase, al mismo tiempo, recuperar desde la TSD (Teoría de las Situaciones Didácticas) el impacto que tiene un acercamiento formal sobre el contenido de fracciones con alumnos de $1^{\circ}$ grado de primaria, aprendizaje que actualmente no se encuentra en el currículo oficial para primer grado. El enfoque cualitativo que se utilizó permite una interpretación oportuna ante las particularidades que se ponen de manifiesto en la práctica docente, recuperada a través de una videograbación que permite visualizar las relaciones y conexiones dadas en la clase. Para realizar la tarea, se toma como base la estrategia de María Bertely (2000) al trabajar la recuperación de la práctica por medio de los registros de clase (simple y ampliado). 


\section{Resultados}

Analizar la práctica educativa ha de conducir a la mejora más allá del aspecto personal, es un punto de partida para generar conciencia acerca de elementos que se deben mantener y fortalecer, así como aquellos que precisan de una revaloración y replanteamiento. En este sentido, se ponen de manifiesto las distintas fases de la Teoría de las Situaciones Didácticas, recuperando en cada una de ellas, las particularidades de la intervención docente y los resultados que se desprenden. Para iniciar, se preparó el medio con una propuesta sencilla que a partir de las actividades que los alumnos practican de manera cotidiana, les resulta fácil de representar.

Ma: Van a cortar una hoja y la van a compartir con el compañero que está a tras de ustedes.

Ao: Te voy a dar del mismo tamaño./Los alumnos comienzan a cortar la hoja, hacen un doblez por mitad, cortan con sus tijeras y hacen la entrega correspondiente/

Ma: vamos a suponer que les doy una naranja, esa naranja se la van a comer en dos momentos, un momento es la hora del receso y el otro momento es la hora de la fruta (escribe en el pizarrón).

Ao: o sea, la vamos a partir, una nos la vamos a comer en el receso y otra en la hora de la fruta.

Ma: eso es en lo que se van a poner a pensar. En su hoja, escriban receso y dibujen lo que se van a comer en la hora del receso, escriben hora de la fruta y dibujan lo que se van a comer a esa hora.

La apertura de la sesión se da con la recuperación de conocimientos previos: una fruta que deben consumir en dos momentos distintos, dando muestra así del subdominio del conocimiento del tema KoT, pues reconoce el reparto de una naranja como un contexto claro para la representación de fracciones de reparto en una magnitud continua. La generalidad recuperada está en función de dos dibujos similares en proporción. Atendiendo la didáctica de la TSD, esta actividad no se validó, por lo tanto no hubo correcciones ni autocorrecciones por parte del grupo, hasta el momento.

\section{Fase de acción.}

Una vez recuperadas las nociones iniciales de los alumnos, se entabló una conversación para contextualizar y proceder con la emisión de la consigna.

Ma: Un personaje que se llama "El come galletas"

Ao: eeee, ya sé quién es... el de Elmo...

Ma: Diego, ¿Cómo es el come galletas?

Diego: Es así como Elmo, es azul y nomás come galletas.

/la maestra saca unas galletas y los niños se muestran contentos/

Ma: El come galletas les mandó una galleta para que la compartan con uno de sus compañeros, será el mismo con el que ya compartieron la hoja. Quiero que la compartan, no se la vayan a comer todavía.

El momento refleja uso de material atractivo para los alumnos, tal como fue el personaje infantil que demostraron conocer muy bien los alumnos, así mismo las galletas y el material didáctico, poniendo de manifiesto que se conoce al grupo, en palabras del modelo MTSK, se manifiesta el subdominio KFLM (características de aprendizaje), pues se tiene conocimiento de los intereses que tienen, así como de los recursos llamativos que abren la posibilidad de construir significado de las actividades.

Se continuó entregando una hoja de trabajo para recortar el dibujo de una galleta y repartirla como lo hicieron con la galleta real; la forma de proceder se desprendió de la siguiente consigna:

Ma: /con la hoja de trabajo pegada en el pizarrón/ Lo mismo que hicieron con la galleta que les entregué, van a hacerlo ahora con esta galletita de papel. Lo van a hacer todos, no sólo a los que les di galleta. 
Ma: aquí dice nombre (debajo de los platos), no le vamos a poner nuestro nombre. Lo que le vamos a poner es el nombre de la parte que le tocó a tu compañero, y el nombre de la parte que te tocó a ti, ¿Cómo se llama esa parte que le tocó de la galleta?

\section{Ma: Alison, ¿qué vamos a hacer?}

Alison: A repartir la galleta con nuestro compañero.

Aos: recortarla, ponerle nombre...

Un punto importante que se refleja en las líneas anteriores es la devolución de la consigna, importante para verificar la comprensión que el grupo ha tenido de ésta, al mismo tiempo que se da la comunicación alumno-alumno que muchas veces da lugar a un mayor entendimiento de lo que se va a realizar; en la misma línea se hace presente el subdominio del conocimiento de las características del aprendizaje (KFLM), puesto que la profesora reconoce las posibilidades de que el alumnado esté construyendo ideas erróneas sobre las actividades sugeridas, recurriendo a la devolución como estrategias para contrarrestar dichas dificultades.

Una vez que los alumnos se encuentran actuando sobre el material, cobra sentido los momentos a-didácticos, donde la participación del docente se caracteriza por atribuir la responsabilidad del conocimiento en los alumnos sin desaparecer del escenario, sino monitoreando el trabajo y participando especialmente por medio de devoluciones para conservar el rumbo de la sesión.

Ao: Ya terminé de recortar.

Ma: ¿qué sigue?

Ao: Repartir, /levanta el recorte y las tijeras/. ¿Así le recortamos?

Ma: Piénsenle muy bien, ¿cómo le van a recortar para ustedes y para su compañero?

\section{$[\ldots]$}

Ma: Se termina el tiempo.

\section{Fase de formulación/ Fase de validación}

La revisión del producto se realiza en grupo, atendiendo las fases de formulación y validación. En primer lugar, se solicitó a los alumnos que tenía un reparto desigual y/o escribieron nombres no convencionales a la fracción de galleta que dieron a cada persona.

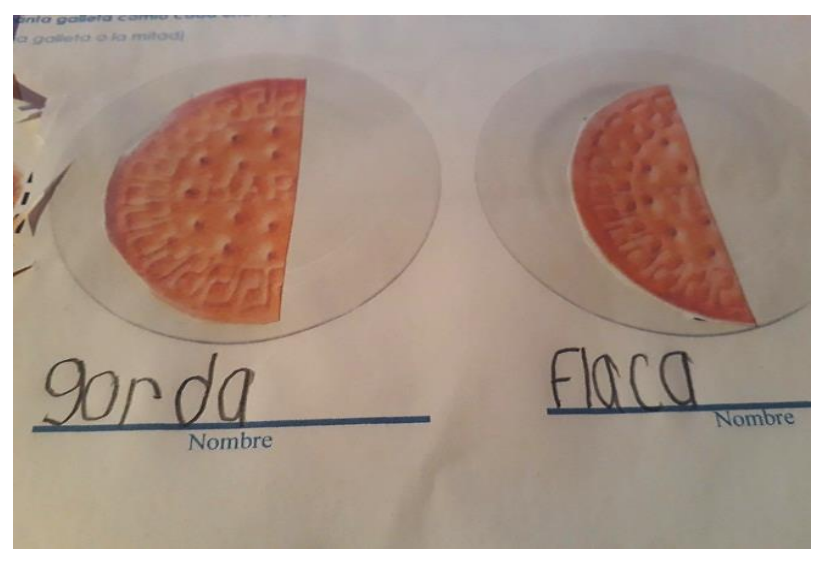

Figura 2 Conceptos a los repartos

Fuente: elaboración propia

$10 \%$ del grupo realizó procedimientos como este donde el reparto representa partes desiguales, además de referirse a ellas con un nombre no convencional en el tema de fracciones.

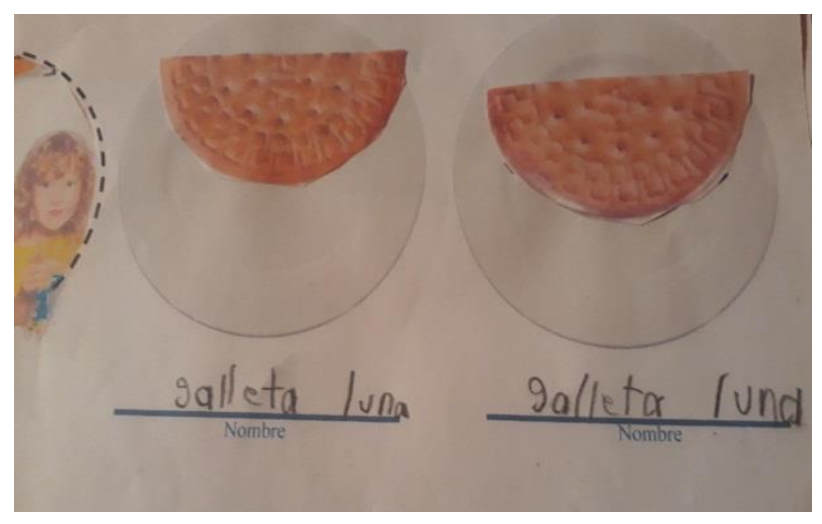

Figura 3 Reparto convencional

Fuente: elaboración propia

Para el presente resultado obtenido en el $50 \%$ de los trabajos, reparto convencional, nombre no convencional, se formula: 


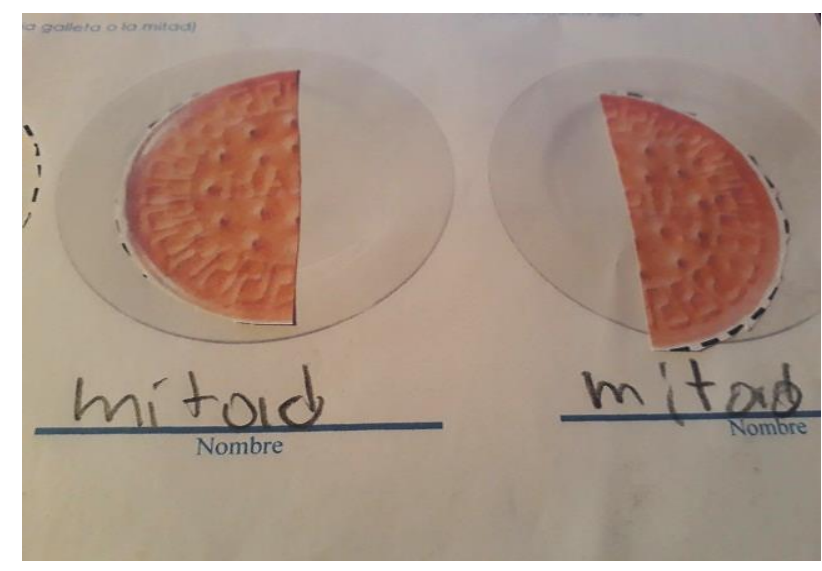

Figura 4 División parte-todo Fuente: elaboración propia

Recorté la galleta, la doblé hasta que quedaron igualitas y de ahí quedó una línea y de ahí la recorté. Por otro lado, 40\% del grupo, realizó una división parte-todo (1/2) refiriéndose a ellas con la palabra "mitad".

Ao: la doblé y la corté.

Ma: ¿cómo le escribiste?

Ao: mitad y mitad, porque yo tengo una mitad y él otra mitad.

La dinámica continuó para desarrollar una segunda consigna:

\section{/Diego lee en la hoja de trabajo/}

Diego: Kevin y sus amigos ya cortaron sus rebanas de pizza, pero quieren saber qué pasaría si las vuelven a juntar. Recorta las rebanadas y forma nuevamente la pizza en el plato y qué pasa.

\section{Ma: ¿Qué vamos a hacer Santigo?}

Santiago: Vamos a recortar los pedazos y vamos a pegarlos en el plato.

Ma: Vamos a pegarlos en el plato, tratando de formar la pizza completa.

Axel: Antes de pegarla voy a intentar a ver si queda.

Aos: Yo también...
Una vez más se dio lugar a la presencia de la fase de acción donde los alumnos trabajaron de forma autónoma, los momentos adidácticos estuvieron en función de que el alumnado reflejara resultados con respecto a la conformación de la unidad; poniendo en escena el subdominio de conocimiento de la práctica (KPT) se formularon y validaron los procedimientos, entre ellos el siguiente:

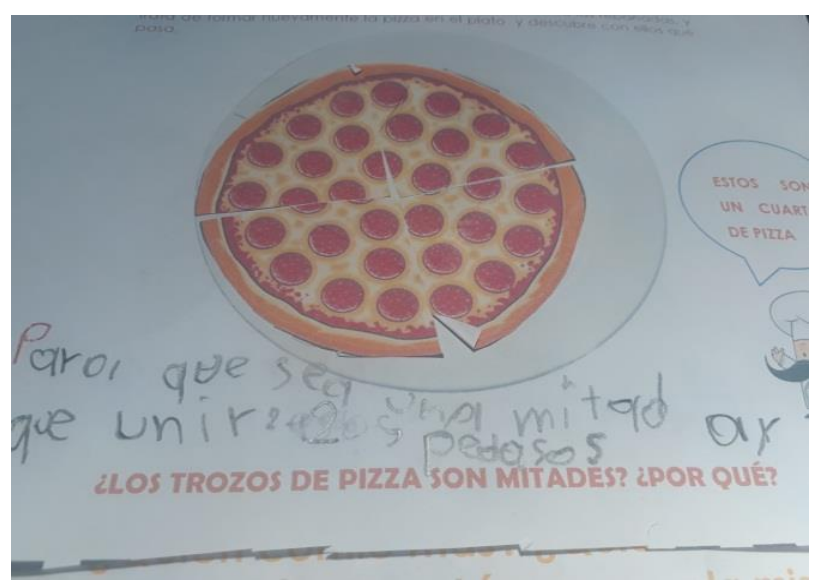

Figura 5 Mitades

Fuente: elaboración propia

En la fase de formulación se planteó la pregunta, ¿uno de esos pedazos representa la mitad de la pizza? En las primeras participaciones mencionaron que sí, que cada pedacito es una mitad, que todas son mitades, mientras que Martín mencionó lo que se muestra en la Figura 5, "para que sea una mitad, hay que unir dos pedazos". Esto muestra las construcciones que lograron hacer los alumnos pues la controversia generada en la fase de formulación/validación, logró concretarse en conclusiones significativas como la de Martín, fue aceptada por el grupo, al mismo tiempo que se dio la corrección y autocorrección de las nociones personales.

Las evidencias, muestran los alcances del alumnado, el material que empleó en el desarrollo del contenido: galletas y posteriormente hojas de trabajo interactivas donde los alumnos manipulan las representaciones (galleta y pizza), esto atiende las características de aprendizaje según Piaget y Inhelder (1976); los alumnos de primer grado están entre seis y siete años de edad, lo que los ubica en el tránsito de la etapa de operaciones preoperacionales a las operaciones concretas; comienzan a construir conclusiones válidas a partir de la lógica, siempre y cuando se parta de situaciones concretas. 
Identificamos el conocimiento de características de aprendizaje (KFLM) que a su vez se relaciona con el conocimiento de la enseñanza (KMT) al recuperar el uso de materiales y recursos en el desarrollo del tema.

\section{Institucionalización.}

Para cerrar la sesión, de acuerdo con la TSD, se concreta de manera formal el trabajo con la intervención del docente en función de sintetizar conceptos y clarificar el contenido.

Ma: Con la galleta vimos que la mitad es partirla en dos partes; a mí me tocó un medio de la galleta y a mi compañero le tocó otro medio de la galleta, es decir, tenía la galleta (dibuja la galleta en el pizarrón) un medio para mí y un medio para mi compañero, que es lo mismo a decir media galleta. Cuando estamos hablando de fracciones, lo correcto es decir, a mí me tocó media galleta y a mi compañero media galleta, es más claro que decir, me tocó una luna. Lo apropiado es decir un medio, media galleta....

Ma: Al pasar a los trozos de la pizza, ya no había dos pedazos ¿verdad? ¿cuántos había?

\section{Aos: Cuatro}

Ma: Cuatro trozos (los dibuja en el pizarrón), estos dos formarán la mitad de la pizza y estos dos formarán la otra mitad. A este pedacito se le llama como dice el monito, ¿qué está diciendo?

Aos: Estos son un cuarto de pizza.

Ma: Este trocito se llama un cuarto, ¿entonces cómo se llama otro pedacito?

Bertín: El otro cuarto.

Ma: si corto la pizza en cuatro partes ¿cuántos cuartos tiene?

\section{Aos: Cuatro.}

/La maestra hace recortes de la pizza: un medio y dos cuartos para la comparación de porciones/
Se hicieron algunos ejemplos de uso de los conceptos aprendidos al beber medio vaso de agua, debemos servirlo a la mitad, si queremos un cuarto de la naranja, debemos partirla en cuatro partes y comer una de ellas. El alumnado contribuyó con algunos ejemplos similares, comer medio plátano, medio jugo, etc. La maestra menciona de forma clara las conclusiones del trabajo durante la sesión, a través de un lenguaje claro que permita a los alumnos comprender los conceptos que para la mayoría de ellos, son nuevos. La incorporación de preguntas dentro de la fase de institucionalización se debe a las características del grupo (KFLM) pues la maestra reconoce las posibles ideas erróneas y/o parciales que los educandos se pueden crear, por lo que amerita las aportaciones directas de la maestra cuestionando para reafirmar la comprensión que se logra. Para evidenciar de manera concreta las conclusiones del presente análisis, es pertinente exponer el siguiente esquema como representación gráfica de las relaciones en la práctica docente que tuvieron lugar durante la sesión trabajada.

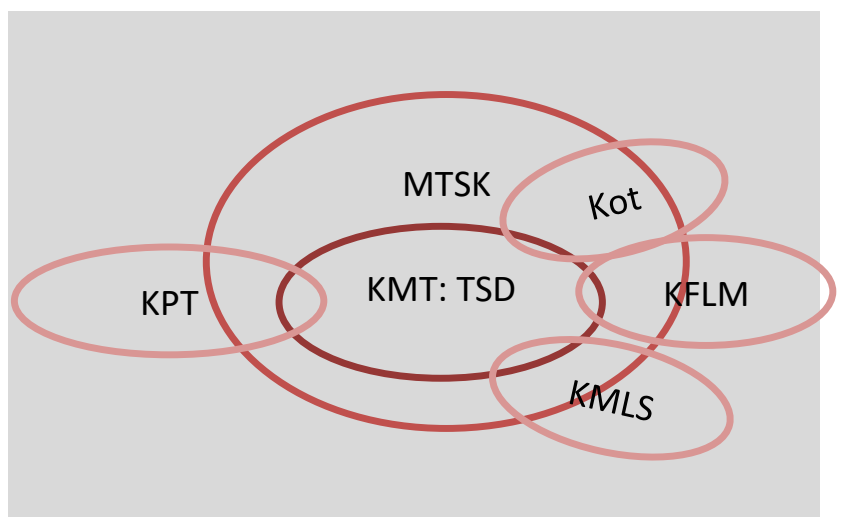

Figura 6 Esquema de relaciones

Fuente: elaboración propia

La Figura 6 refleja las relaciones encontradas a partir del análisis durante la sesión de clases. El modelo MTSK es el centro del conocimiento del profesor que deriva los subdominios; el conocimiento de la enseñanza (KMT) cobra un papel importante, ya que la sesión se desarrolla bajo la teoría de enseñanza TSD, con ella se relaciona el subdominio del conocimiento de las características de aprendizaje (KFLM), pues durante las fases tanto en planeación como en ejecución, se toman en cuenta las aptitudes, dificultades y etapas de desarrollo que presentan los alumnos. 
En la misma línea se interviene con conocimiento de los estándares de aprendizaje (KMLS), cimentando bases de contenidos que trabajarán en ciclos posteriores. Por otro lado, dentro de la situación didáctica encontramos el conocimiento de la práctica (KPT); la fase de validación da lugar de forma inherente a este subdominio con el contraste de resultados, procesos de resolución, entre otros. Así mismo el KoT, se conoce el tema, los conceptos a trabajar, los procedimientos, centrando la mirada en la contextualización de los problemas.

\section{Conclusiones}

El primer acercamiento formal de los alumnos a contenidos de fracciones está en función a la propuesta oficial del plan y programas de estudio en educación básica, específicamente en primaria, donde se señala el aprendizaje esperado que se ha de alcanzar en tercer grado de primaria, "Usa fracciones con denominador hasta 12 para expresar relaciones parte-todo, medidas y resultados de reparto" (SEP, 2017, pág. 236), sería bueno preguntarnos, ¿por qué no trabajar fracciones en primer grado de primaria? como parte introductoria a lo que se dicta trabajar en tercer grado, puede ser tal vez, una manera de contrarrestar las dificultades que actualmente representa el estudio de las fracciones que los alumnos de educación primaria manifiestan.

Los resultados expresan que un conocimiento acertado por parte del profesor ha de generar resultados satisfactorios en los alumnos, en medida que el docente amplía su conocimiento del contenido matemático y su didactificación, mayores son los alcances de una intervención didáctica. En este análisis tenemos presente, ante todo el subdominio de conocimiento de la enseñanza (KMT), ya que se trabaja la sesión bajo una de las teorías de enseñanza institucionalizada: TSD, respetando las fases y momentos, así como las características que cada uno de ellos tiene de manera implícita: las consignas, las devoluciones, momentos didácticos y adidáctico, etc.

El subdominio del conocimiento didáctico del contenido (PCK) se encontró con mayor frecuencia, ya que el diseño y desarrollo de la sesión se da con base a las características del aprendizaje.
Lo que generó la consolidación de conceptos convencionales sobre fracciones (medios y cuartos) en alumnos de primer grado de primaria. Los resultados aquí obtenidos pueden ser considerados para el replanteamiento de aprendizajes esperados del currículo, ya que alumnos del primer ciclo demuestran ser capaces de construir de manera formal, conceptos referentes al tema de fracciones desprendidos del contexto; sería importante habituarnos desde una temprana edad al uso convencional de dichos conceptos, lo que puede generar un cambio social en cuanto a la familiarización que se tiene con los términos y uso de números fraccionarios.

\section{Referencias}

Bertely, Busquets, M. (2000) La escritura etnográfica: ¿desde dónde y para quién?, en Taller: IX Simpo-sio Interamericano de Investigación Etnográfica en Educación. México, Universidad Pedagógica Nacional/Ajusco.

Brousseau, G. (2007). Iniciación al estudio de la teoría de las situaciones didácticas. Argentina: Ministerion de educación.

Llinares, S., \& Sánchez, M. V. (1997). Fracciones. España: Síntesis.

Carrillo, J., Montes, M., Contreras, L. C., \& Climent, N. (2017). El conocimiento del profesor desde una perspectiva basada en su especialización: MTSK. Annales de Didactique et de Sciences Cognitives, 22, 185-206.

Perales, R. (2006). La significación de la práctica. México.

Piaget, J. \& B. Inhelder (1976). Génesis de las estructuras lógicas elementales. Buenos Aires: Guadalupe.

SEP. (2017). Aprendizajes clave para la educación integral. Ciudad de México: SEP. 